\title{
Presión percibida para el consumo de alcohol y variables de resistencia en la adolescencia
}

\section{Perceived pressure for drinking alcohol and endurance variables in adolescence}

\author{
María del Carmen Pérez-Fuentes ${ }^{1}$, África Martos Martínez ${ }^{2}$, María del Mar Molero Jurado ${ }^{3}$, Ana Belén \\ Barragán Martín ${ }^{4}$, María del Mar Simón Márquez ${ }^{5}$ Rosa María del Pino Salvador ${ }^{6}$ \\ ${ }^{1}$ Universidad de Almería; Universidad Politécnica y Artística del Paraguay, Asunción (Paraguay) mpf421@ual.es \\ ${ }^{2}$ Universidad de Almería amm521@ual.es \\ ${ }^{3}$ Universidad de Almería mmj130@ual.es \\ ${ }^{4}$ Universidad de Almería abm410@ual.es \\ ${ }^{5}$ Universidad de Almería msm112@ual.es \\ ${ }^{6}$ Universidad de Almería dsr333@ual.es
}

Recibido: $10 / 9 / 2020$

Aceptado: 18/12/2020

Copyright $(\mathrm{C}$

Facultad de CC. de la Educación y Deporte.

Universidad de Vigo

Dirección de contacto:

María del Carmen Pérez Fuentes

Edificio Departamental de Humanidades y

Ciencias de la Educación I (Edificio A)

Planta 1, Despacho 060

Universidad de Almería

Ctra. Sacramento, $\mathrm{s} / \mathrm{n}$

La Cañada de San Urbano

04120 Almería

\section{Resumen}

La adolescencia es una etapa caracterizada por la experimentación de cambios y nuevas experiencias que, a veces, pasan a ser conductas de riesgo, tales como el consumo de alcohol. Actualmente, la implicación de los adolescentes en este tipo de experiencias va en aumento, generando consecuencias negativas en el desarrollo del adolescente. Alguno de los factores que promueve el inicio en el consumo de bebidas alcohólicas es la presión percibida por el grupo de iguales hacia el consumo. Por tanto, los objetivos del presente estudio son analizar la asociación entre diferentes variables individuales como la autoestima, la impulsividad, la sensibilidad a la ansiedad y las expectativas hacia el consumo de alcohol, así como su implicación en la resistencia a la presión percibida para consumir alcohol en una muestra de 1.287 alumnos con edades comprendidas entre 14 y 18 años. Para ello, se utilizó el Cuestionario Resistencia a la Presión de Grupo en el Consumo de Alcohol, Cuestionario de Expectativas del Alcohol en Adolescentes, Anxiety Sensitivity Index-3, Rosenberg's Self-Esteem Scale y Barratt Impulsiveness Scale-11-A. Los resultados mostraron la existencia de asociaciones positivas entre la presión percibida y las expectativas positivas hacia el consumo, la sensibilidad a la ansiedad física, cognitiva y social y con la impulsividad general. De este modo, es importante seguir con esta línea de investigación y desarrollar estrategias de intervención innovadoras, con el objetivo de reducir las conductas de riesgo en los adolescentes.

\section{Palabras clave}

Presión Percibida, Adolescencia, Sensibilidad a la Ansiedad, Autoestima, Expectativas de Consumo

\section{Abstract}

Adolescence is a stage characterized by experimentation with changes and new experiences, which at times go through risk behaviors, such as drinking alcohol. At 
present, involvement of adolescents in this type of experiences is on the rise, generating negative consequences in adolescent development. Some of the factors that promote the start of drinking alcohol is perceived peer group pressure to drink. Therefore, the objectives of this study were to analyze the association between different individual variables, such as self-esteem, impulsivity, sensitivity to anxiety and expectations for drinking alcohol, as well as their involvement in resistance to perceived pressure to drink alcohol in a sample of 1.287 students aged 14 to 18 . The Cuestionario Resistencia a la Presión de Grupo en el Consumo de Alcohol [Resistance to Group Pressure to Drink Alcohol Questionnaire], Cuestionario de Expectativas del Alcohol en Adolescentes [Adolescent Expectations for Alcohol Questionnaire], Anxiety Sensitivity Indes-3, Rosenberg's Self-Esteem Scale and Barratt Impulsiveness Scale-11-A. The results showed the existence of positive associations between perceived pressure and positive expectations for drinking, sensitivity to physical, cognitive and social anxiety and with general impulsivity. Thus, it is important to continue with this line of research and develop innovative intervention strategies for reducing adolescent risk behaviors.

\section{Keywords}

Perceived Pressure, Adolescence, Sensitivity to Anxiety, Self-Esteem, Expectations for Use

\section{INTRODUCCIÓN}

La adolescencia es una etapa vulnerable caracterizada por la búsqueda de nuevas sensaciones, como es el consumo de sustancias, entre las que se encuentra el alcohol (González-Yubero, Palomera y Lázaro-Visa, 2019; Jiménez, 2011; Plan Nacional Sobre Drogas, 2016; Pons y Buelga, 2011). Según la última Encuesta Estatal sobre Uso de Drogas en Enseñanzas Secundarias (DGPNSD, 2018), el 76,9\% de los estudiantes de Enseñanzas Secundarias Obligatorias con edades comprendidas entre los 14 y 18 años, reconoce haber consumido bebidas alcohólicas alguna vez, mostrando que el alcohol es la sustancia psicoactiva más extendida entre esta población. Esta motivación a la hora de consumir alcohol está influenciada por la facilidad de acceso, así como por la presión percibida por parte del grupo de pares y por las consecuencias sociales satisfactorias que derivan de la ingesta de alcohol, tales como la obtención de refuerzos por parte de los iguales (Pons y Buelga, 2011). Sin embargo, se ha comprobado que, ya en el período de la adolescencia, existen altas expectativas positivas hacia el alcohol, de manera que son los jóvenes que consumen de manera más abusiva, los que asocian este consumo a un mayor número de consecuencias positivas al alcohol (Pedreira, Blanco, Pérez-Chacón y Quirós, 2014).

Por ello, es en este periodo donde el joven se siente partícipe de un grupo de iguales, con los que comparte valores, espacios, reglas, conductas y modas. Estos grupos tienen una gran influencia en los adolescentes y, por ello, presentan: a) una serie de normas que el adolescente debe seguir, b) compromisos que el joven debe aceptar y, c) valores que van a ayudar a la construcción de su propia identidad (Calafat, García, Juan, Becoña y Fernández-Hermida, 2014; Riquelme, García y Serra, 2018; Veiga, García, Reeve, Wentzel y García, 2015). Dentro de estos grupos de iguales, existe un hecho que llega a formar parte de la cultura de los mismos: el consumo de alcohol, el cual afecta a cualquier grupo de edad. Además, este tipo de consumo implica un espacio compartido, 
simbólico e interactivo que lo promueve (Álvarez, Alonso y Guidorizzi, 2010; Committee on National Alcohol Policy and Action, 2014; Pons y Buelga, 2011).

Por otro lado, cabe destacar que, actualmente, los adolescentes pasan la mayor parte de su tiempo con su grupo de iguales, convirtiéndose éstos en una fuente de afecto y comprensión (Rodríguez, Perozo y Matute, 2014). En este sentido, se observa la evidente influencia que ejercen los iguales en la ingesta de alcohol, debido a que, según Rodríguez et al. (2014), los adolescentes comienzan a consumir alcohol motivados por la conducta de sus amigos y en busca de un refuerzo social. Por ello, la presión ejercida por el grupo es considerada como un medio que utilizan los pares para incitar al sujeto a consumir y, la resistencia a esta presión es la capacidad del sujeto para abstenerse. En este sentido, cabe destacar los resultados de la investigación de Londoño y Valencia (2010), sobre la asociación entre las expectativas sobre la ingesta de alcohol y el manejo a la presión de grupo, donde se muestra cómo aquellos adolescentes que poseen poca capacidad para abstenerse a los deseos de sus pares, tienen mayor riesgo de consumo y viceversa.

\subsection{Variables individuales y consumo de alcohol}

Varios estudios corroboran que el consumo de alcohol en la etapa adolescente está relacionado con variables individuales, como son la autoestima (Álvarez et al., 2010), actitudes e influencias de los amigos hacia el alcohol (Heimisdottir, Vilhjalmsson, Kristjansdottir y Meyrowitsch, 2010; Llorens, Barrio, Sánchez, Suelves y ESTUDES Grupo de Trabajo, 2011), rasgos de personalidad como la impulsividad (Koob y Volkkow, 2010), la sensibilidad a la ansiedad y la permisividad, entre otras (PérezFuentes et al., 2015; Teixidó-Compañó et al., 2019).

Entre los factores personales relacionados con el consumo, se puede encontrar que la autoestima es uno de ellos, ya que constituye una variable psicológica multidimensional del adolescente que actúa de mediador entre variables familiares y problemas de conducta (Maganto, Peris y Sánchez, 2019). Algunos autores señalan que la autoestima es una herramienta de protección que contribuye a proteger el bienestar biológico, social y mental de la persona ante problemas psicológicos (Álvarez et al., 2010). Es decir, tener una alta autoestima es estimado como una variable protectora y, sobre todo, fundamental en la adolescencia. Esto se debe a que, según Álvarez et al. (2010), presentar una autoestima baja puede transformase en una variable de riesgo a la hora de iniciarse en el consumo de bebidas alcohólicas. En este sentido, cuando un sujeto presenta baja autoestima y mayor desajuste psicológico, puede incluso llegar a desarrollar conductas violentas, siendo más vulnerable, así, su grupo de iguales puede ejercer una mayor presión hacia el consumo, incitándolo a ello (Pérez-Gramaje, García, Reyes, Serra y García, 2019; Rodríguez et al., 2014).

Otro de los factores individuales relacionados con el consumo de bebidas alcohólicas es la impulsividad (Riaño, Guillén y Buela, 2015; Martínez-Loredo et al., 2015). Diversos estudios muestran cómo el consumo de alcohol está ligado con las conductas violentas, agresivas e impulsivas (Gázquez, Pérez-Fuentes, Carrión y Santiuste, 2009; Pérez-Fuentes et al., 2015; Ribas et al., 2015; Sanabria y Uribe, 2009). Por ello, los adolescentes con conductas agresivas muestran alta impulsividad y niveles bajos de autocontrol, convirtiéndose la primera en una patología muy común en los adolescentes (Contreras, Molina y Cano, 2012; Moñino, Piñero, Arense y Cerezo, 2013; Pérez- 
Fuentes y Gázquez, 2010; Pérez-Fuentes et al., 2015). Además, se ha encontrado que la presencia de impulsividad en los jóvenes se caracteriza por una tendencia mayor al consumo (Narváez y Caro, 2015). En conclusión, la impulsividad se puede definir como la tendencia a llevar a cabo acciones concretas sin evaluar previamente las consecuencias que puedan originarse (Adan, 2012). De esta manera, este factor puede ser una consecuencia derivada del consumo (Fuentes, Alarcón, García y Gracia, 2015; Pérez-Fuentes et al., 2015).

Finalmente, otro de los factores que hay que tener en cuenta es la sensibilidad a la ansiedad. Para Molina, Sandín y Chorot (2013) y Sánchez (2009), la sensibilidad a la ansiedad (SA) es una variable que define el miedo a los síntomas que ésta pueda provocar por diferentes causas, ya que se debe a la creencia de que la ansiedad origina consecuencias negativas y peligrosas para el propio sujeto. En este sentido, cabe destacar que la sensibilidad a la ansiedad y el consumo de sustancias están estrechamente relacionadas, ya que, a mayor consumo y dependencia al alcohol, existe una mayor puntuación de sensibilidad a la ansiedad (Valdez y Lira, 2013). Por tanto, teniendo en cuenta la asociación existente entre la sensibilidad a la ansiedad y el consumo (Pérez-Fuentes, Molero y Gázquez, 2019), es esencial resaltar la prevención de esta conducta de riesgo.

En definitiva, según los resultados de Moral, Rodríguez y Sirvent (2006), las creencias erróneas sobre los efectos del consumo de alcohol y la escasa resistencia a la incitación del grupo de iguales están relacionadas con comportamientos orientados al consumo.

\subsection{Objetivo}

El objetivo de este trabajo fue analizar la relación entre diferentes variables individuales como la autoestima, la impulsividad, la sensibilidad a la ansiedad y las expectativas hacia el consumo de alcohol, así como su implicación en la resistencia a la presión percibida para consumir alcohol.

\section{MÉTODO}

\subsection{Población y Muestra}

La muestra del presente estudio estuvo compuesta por un total de 1.287 alumnos de Educación Secundaria Obligatoria pertenecientes a diferentes centros de la provincia de Almería (España), todos ellos de carácter público. Las edades de los alumnos estuvieron comprendidas entre 14 y 18 años, presentando una media de edad de 15,11 años $(D T=0,91)$. En relación a la distribución por sexo, el $47,1 \%(n=606)$ eran hombres y el $52,9 \%(n=681)$ mujeres, con 15,12 años $(D T=0,94)$ y $15,10(D T=0,88)$ años de media, respectivamente. El cuanto a la distribución por curso el $55 \%(n=707)$ asistían a $3^{\circ}$ E.S.O, y el $45 \%(n=577)$ asistían a $4^{\circ}$ curso. 


\subsection{Instrumentos}

Se utilizó un cuestionario elaborado ad hoc para la recogida de datos sociodemográficos (edad, sexo, curso), cuestiones relacionadas con el consumo de alcohol (sí/no), así como con la frecuencia (desde $0=$ He tomado bebidas alcohólicas muy pocas veces en mi vida, hasta 5=Muchas veces al día).

Cuestionario Resistencia a la Presión de Grupo en el Consumo de Alcohol (CRPG) (Londoño, Valencia, Sánchez y León, 2007). Está compuesto por un total de 45 ítems los cuales miden el manejo de la presión de grupo para el consumo de alcohol. Las opciones de respuesta siguen una escala tipo Likert de 4 puntos (desde 1= nunca, hasta 4 = siempre). Así, el cuestionario está formado por tres factores: resistencia a la presión de grupo directa incluyendo los ítems del 1 al 18 (Te ves obligado a beber porque te es difícil decir NO), resistencia a la presión de grupo indirecta incluyendo los ítems del 19 al 40 (Es más deseable que tú), resistencia a la presión percibida incluyendo los ítems del 41 al 45 (Cuando tus amigos se burlan de ti porque no deseas beber). La fiabilidad de cada una de las escalas fue de $\alpha=0,69, \alpha=0,85$, y $\alpha=0,81$, respectivamente.

Cuestionario de Expectativas del Alcohol en adolescentes - adaptación al español (Gázquez et al., 2015). Está compuesto por 7 ítems los cuáles evalúan las creencias sobre los efectos positivos/negativos del alcohol en la conducta social y emocional de los adolescentes. Las diferentes opciones de respuesta siguen una escala Likert de 5 puntos (desde totalmente en desacuerdo, hasta totalmente de acuerdo). El cuestionario está formado por dos escalas: Expectativas positivas (Normalmente el alcohol tiene efectos muy positivos sobre las personas) y Expectativas negativas (El alcohol empeora el razonamiento y la coordinación de las personas).

Anxiety Sensitivity Index-3 (ASI-3) (Peterson y Reiss, 1992). Se empleó la versión española del cuestionario (Sandín, Valiente, Chorot y Santed, 2007). Está formado por un total de 18 ítems, las opciones de respuesta siguen una escala tipo Likert de 5 puntos (desde $0=$ Nada o casi nada, hasta $4=$ Muchísimo). En este cuestionario se pueden encontrar 3 escalas: Sensibilidad Física (Me asusto cuando mi corazón late de forma rápida), Sensibilidad cognitiva (Cuando no puedo mantener mi mente concentrada en una tarea, siento la preocupación de que podría estar volviéndome loco/a) y Sensibilidad social (Para mí es importante no dar la impresión de estar nervioso/a). La fiabilidad de cada una de las escalas fue de $\alpha=0,85, \alpha=0,84, \mathrm{y} \alpha=0,77$, respectivamente.

Rosenberg's Self-Esteem Scale (Rosenberg, 1965). Consta de un total de 10 ítems los cuales evalúan la satisfacción de uno mismo en una escala tipo Likert de 4 puntos desde 1 (Totalmente de acuerdo) hasta 4 (Totalmente en desacuerdo) (Me siento una persona tan valiosa como las otras). Así bien, la fiabilidad de este instrumento fue $\alpha=$ 0,86 .

Barratt Impulsiveness Scale-11-A (BIS-11 ${ }^{\text {a }}$ (Cosi, Vigil-Colet, Canals y LorenzoSeva, 2008). Concretamente, se empleó la versión adaptada para adolescentes españoles (Martínez-Loredo et al., 2015). Este instrumento consta de 30 ítems los cuales evalúan la impulsividad general. Las opciones de respuesta siguen una escala tipo Likert de 4 puntos (desde 1 "rara vez o nunca" hasta 4 "Siempre o casi siempre"). En este cuestionario se agrupa en dos factores: Impulsividad general (Hago las cosas sin pensarlas) e Impulsividad no planificada (Planifico lo que tengo que hacer). La 
fiabilidad para el factor de impulsividad no planificada fue $\alpha=0,63$ y para la impulsividad general, la fiabilidad fue $\alpha=0,76$.

\subsection{Procedimiento de recogida y análisis de datos}

En primer lugar, se procedió a realizar una reunión informativa con la finalidad de explicar al centro los objetivos de la presente investigación, garantizando así el tratamiento confidencial de los datos. Seguidamente, se procedió a realizar la implementación de los cuestionarios en los diferentes centros educativos seleccionados, siendo la implementación de las pruebas en el aula habitual donde el alumnado recibe la docencia, contando con la presencia del profesor/tutor correspondiente. Antes de comenzar, se les explicaron de manera detallada las instrucciones al alumnado y se garantizó el carácter anónimo de sus respuestas y, por tanto, la reserva de su privacidad en el tratamiento estadístico de los datos. Los alumnos cumplimentaron los cuestionarios de manera individual con un tiempo aproximado de 25-30 minutos. En todos los casos, se cumplió de manera estricta con los estándares éticos de la investigación, mediante hoja de consentimiento informado. La investigación cuenta con el informe favorable del Comité de Bioética de la Universidad de Almería (Ref: UALBIO2018/015).

En cuanto al análisis de datos, en primer lugar, con el objetivo de explorar la asociación entre las variables se calculó la matriz de correlaciones bivariadas con el coeficiente de Pearson.

Por otro lado, se realizó un análisis clúster bietápico para determinar los diferentes perfiles, atendiendo a las dimensiones de la sensibilidad a la ansiedad, la impulsividad general, las expectativas positivas hacia el alcohol y la autoestima. Una vez identificados los grupos o clúster, se lleva a cabo un análisis comparativo de medias, para determinar la existencia de diferencias significativas entre los grupos con respecto a la presión percibida, mediante la prueba $t$ de Student para muestras independientes, $y$ el estadístico d de Cohen (1988) para comprobar el tamaño del efecto en las diferencias encontradas, donde los valores inferiores a 0,2 indican un efecto pequeño, 0,5 un efecto medio y 0,8 indica un efecto de alta magnitud.

Para el análisis de los datos se utilizó el paquete estadístico SPSS versión .23 para Windows.

\section{RESULTADOS}

\subsection{Presión percibida: relaciones con la sensibilidad a la ansiedad, la autoestima, la impulsividad y las expectativas hacia el alcohol}

En cuanto a las relaciones entre la presión percibida y la sensibilidad a la ansiedad, tal y como se muestra en la Tabla 1, la presión percibida correlaciona positivamente con los tres tipos de sensibilidad a la ansiedad: factor cognitivo $(r=0,23 p<0,001)$, factor social $(r=0,25, p<0,001)$, y factor físico $(r=0,19 p<0,001)$. Por otro lado, se muestra la existencia de una correlación positiva entre la presión percibida y la impulsividad general $(r=0,18 p<0,001)$. 
Así, en las relaciones entre la presión percibida y las expectativas hacia el alcohol, se muestra cómo la presión percibida correlaciona positivamente con las expectativas positivas hacia el alcohol $(r=0,226, p<0,001)$, sin embargo, no muestra correlación con las expectativas negativas hacia el alcohol $(r=0,02 p=0,42)$. Además, se presenta una correlación de signo negativo entre la presión percibida y la autoestima $(r=-0,125$ $p<0,001)$.

Por último, se presenta un resumen de las puntuaciones medias y desviación estándar de las variables analizadas para la muestra total.

\begin{tabular}{|c|c|c|c|c|c|c|c|c|}
\hline & & $\begin{array}{c}\text { Factor } \\
\text { cognitivo de } \\
\text { ansiedad }\end{array}$ & $\begin{array}{c}\text { Factor } \\
\text { social de } \\
\text { ansiedad }\end{array}$ & $\begin{array}{c}\text { Factor } \\
\text { físico de } \\
\text { ansiedad }\end{array}$ & $\begin{array}{c}\text { Impulsividad } \\
\text { general }\end{array}$ & $\begin{array}{c}\text { Expectativas } \\
\text { positivas }\end{array}$ & $\begin{array}{c}\text { Expectativas } \\
\text { negativas }\end{array}$ & Autoestima \\
\hline \multirow{4}{*}{$\begin{array}{l}\text { Presión } \\
\text { percibida }\end{array}$} & $\begin{array}{l}\text { Correlación } \\
\text { de Pearson }\end{array}$ &, $231 * * *$ &, $257 * * *$ &, $197 * * *$ &, $184 * * *$ &, $226 * * *$ &,$- 125 * * *$ & ,022 \\
\hline & $\begin{array}{c}\text { Sig. } \\
\text { (bilateral) }\end{array}$ & ,000 & ,000 &, 000 &, 000 & ,000 & ,000 & ,429 \\
\hline & $\mathrm{M}$ & 4,74 & 8,19 & 6,90 & 32,45 & 8,28 & 10,73 & 29,36 \\
\hline & DT & 4,98 & 5,20 & 5,61 & 6,85 & 3,01 & 2,49 & 5,90 \\
\hline
\end{tabular}

Tabla 1. Presión percibida, sensibilidad a la ansiedad, autoestima, impulsividad y expectativas hacia el alcohol. Correlación de Pearson

3.2. Perfiles en función de las dimensiones de sensibilidad a la ansiedad, impulsividad general, expectativas positivas hacia el alcohol y autoestima y diferencias en presión percibida

Para la formación de los grupos, se realiza un análisis clúster bietápico con las dimensiones de la sensibilidad a la ansiedad, la impulsividad general, las expectativas positivas hacia el alcohol y la autoestima. A partir de la inclusión de estas variables, resultan dos grupos (Figura 1), con la siguiente distribución: el 71,1\% $(n=774)$ de los sujetos pertenecen al clúster 1 y el 28,3\% $(n=306)$ al clúster 2 .

En la tabla siguiente, se presenta un resumen de las puntuaciones medias y la desviación estándar de las dimensiones de la sensibilidad a la ansiedad, la impulsividad general, las expectativas positivas hacia el alcohol y la autoestima para cada uno de los clústeres.

El primer grupo resultante del análisis clúster (Clúster 1), se caracteriza por presentar puntuaciones más elevadas que la media de la muestra total, en Autoestima. Mientras que, para el factor cognitivo, factor físico, factor social, impulsividad general, y expectativas positivas hacia el alcohol, se obtiene una media por debajo de la encontrada en la muestra total. Por el contrario, en el segundo clúster, las puntuaciones medias obtenidas son inferiores a las de la muestra completa, para la autoestima, mostrando puntuaciones medias superiores en el factor cognitivo, factor físico, factor social, impulsividad general, y expectativas positivas hacia el alcohol, tal y como se observa en la Tabla 2 y en la Figura 1. 


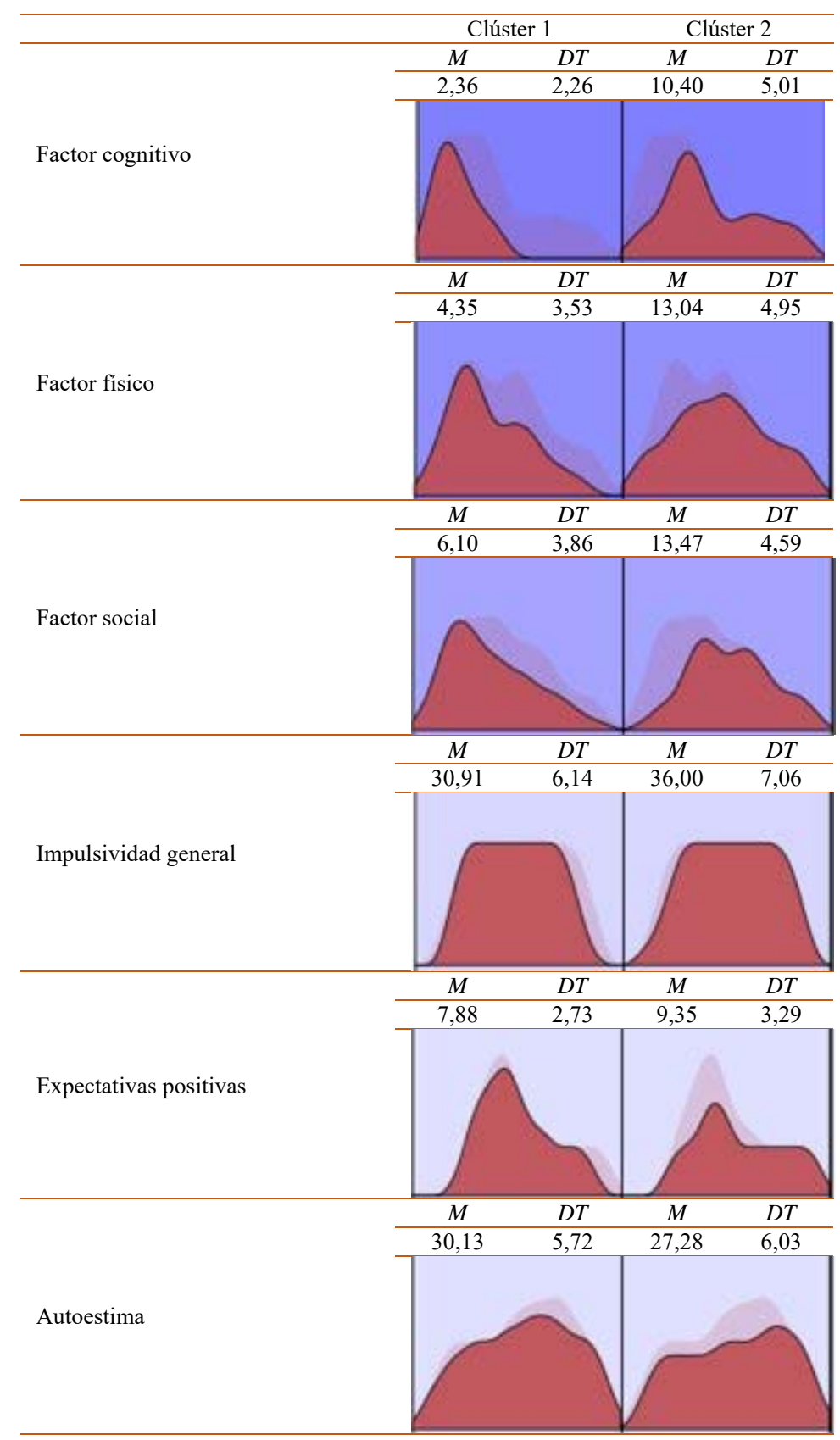

Tabla 2. Composición de los clústeres. Nota. Factores ordenados por importancia de entrada

Tras la clasificación de los grupos, a partir de la solución de dos clústeres, se llevó a cabo una prueba $t$ de Student para muestras independientes, para conocer la existencia de diferencias entre los clústeres con respecto a la presión percibida ante el consumo de alcohol. Así, los resultados muestran cómo existen diferencias significativas entre los clústeres en presión percibida $(t=-8,819 ; p<0.001 ; d=0,60)$, con un tamaño del efecto medio. En este caso se detectan diferencias entre los clústeres, siendo el clúster 2 , el que muestra puntuaciones en presión percibida más elevadas $(M=16,02 ; D T=5,71)$, en comparación con el clúster $1(M=12,98 ; D T=4,66)$. 


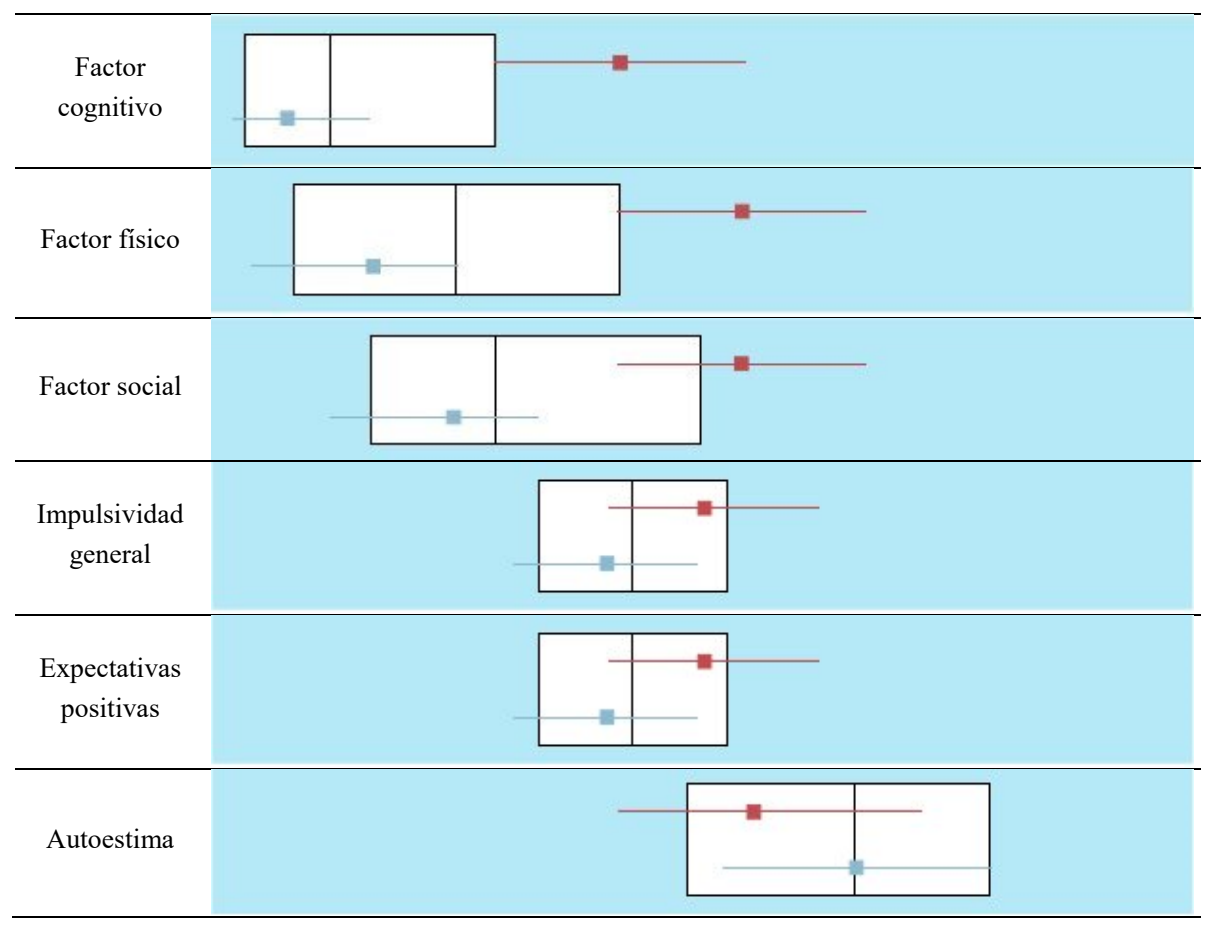

Figura 1. Composición de los clústeres

Tras la clasificación de los grupos, a partir de la solución de dos clústeres, se llevó a cabo una prueba $t$ de Student para muestras independientes, para conocer la existencia de diferencias entre los clústeres con respecto a la presión percibida ante el consumo de alcohol. Así, los resultados muestran cómo existen diferencias significativas entre los clústeres en presión percibida $(t=-8,819 ; p<0.001 ; d=0,60)$, con un tamaño del efecto medio. En este caso se detectan diferencias entre los clústeres, siendo el clúster 2, el que muestra puntuaciones en presión percibida más elevadas $(M=16,02 ; D T=5,71)$, en comparación con el clúster $1(M=12,98 ; D T=4,66)$.

\section{DISCUSIÓN}

Tras analizar los resultados se ha encontrado asociación entre las variables individuales, como la autoestima y las tres dimensiones de la sensibilidad a la ansiedad, la impulsividad y las expectativas hacia el consumo de alcohol y la resistencia a la presión percibida para consumir alcohol. De esta manera y siguiendo las afirmaciones de Londoño y Valencia (2010) y Rodríguez et al. (2014), se puede corroborar que la presión percibida para el consumo de alcohol se correlaciona de manera positiva con la sensibilidad a la ansiedad, la impulsividad y las expectativas positivas hacia el alcohol. Mientras que con la autoestima se correlaciona negativamente.

Por otro lado, los adolescentes pertenecientes al segundo clúster, mostraron una relación existente entre bajas puntuaciones en autoestima y altos niveles de sensibilidad a la ansiedad, impulsividad y expectativas positivas hacia el consumo, al igual que las investigaciones de Álvarez et al. (2010), Pérez-Fuentes et al. (2015) y Rodríguez et al. (2014), en las que se afirma que un sujeto con baja autoestima es más vulnerable, no oponiéndose a los deseos de consumo de bebidas alcohólicas de su grupo de iguales. Además, debido a las características de los sujetos de este clúster, éstos declararon 
puntuaciones en presión percibida elevada por su grupo de iguales, coherente con lo hallado en otras investigaciones (Álvarez et al., 2010; Gázquez et al., 2009; Rodríguez et al., 2014). Al contrario que aquellos pertenecientes al clúster 1 , quienes presentan una relación entre altas puntuaciones en autoestima. Esto puede deberse a que la autoestima funciona como un factor protector frente a las conductas de riesgo en los adolescentes, reduciendo así los niveles de sensibilidad a la ansiedad, impulsividad y expectativas positivas hacia el alcohol. Además, estos sujetos muestran altos niveles de sensibilidad a la ansiedad, impulsividad y expectativas positivas hacia el alcohol bajas (Álvarez et al., 2010; Londoño y Valencia, 2010). Además, cabe destacar que los sujetos del clúster 2 , al tener pocas capacidades para oponerse a los deseos de sus pares, muestran mayores riesgos a la hora de consumir bebidas alcohólicas (Londoño y Valencia, 2010; PérezFuentes y Gázquez, 2010), a diferencia de los sujetos pertenecientes al clúster 1.

En relación con la presión percibida para consumir alcohol, se evidencia que la escasa resistencia a la presión del grupo de iguales se asocia a comportamientos más propensos al consumo, en consonancia con los resultados de los estudios de Moral et al. (2006) y Rodríguez et al. (2014), quienes muestran cómo los adolescentes más vulnerables, se ven más motivados a consumir alcohol, debido a la conducta de sus amigos, siendo incapaces de abstenerse.

Finalmente, y de cara a la aplicación práctica de los datos que aporta el estudio, es importante señalar algunas limitaciones. En primer lugar, el trabajo se centró fundamentalmente en alumnos y alumnas de $3^{\circ}$ y $4^{\circ}$ de Educación Secundaria Obligatoria, lo que podría sesgar la generalización de los resultados a otros cursos de Secundaria. Por lo que para futuras investigaciones sería oportuno analizar todos los cursos de la E.S.O. e incluir otras variables que sean protectoras, como por ejemplo el autocontrol. Por último, la muestra se centró en institutos de Educación Secundaria Obligatoria, sin hacer referencia a adolescentes con absentismo escolar. Por lo que, para posteriores estudios, sería conveniente contar con técnicas de muestreo que nos permitan acceder a este sector de la población.

\section{CONCLUSIONES}

La adolescencia puede traer consigo una serie de conductas de riesgo que dificulten el desarrollo integral de los jóvenes, afectando a la construcción de su propia identidad. La elevada presencia de consumo de bebidas alcohólicas entre los estudiantes de Educación Secundaria supone una grave preocupación a nivel social y académico, debido a las graves consecuencias que puede ocasionar para los jóvenes. Por ello, analizar la relación entre diferentes variables individuales que colaboran en el desarrollo de la persona, como son la autoestima, la impulsividad, la sensibilidad a la ansiedad y las expectativas hacia el consumo de alcohol, así como su implicación en la resistencia a la presión percibida para consumir alcohol, se convierte en un tema de vital relevancia.

Por tanto, siendo el tema tan relevante en el área de Educación, sería aconsejable para el ámbito científico incluir otras variables protectoras ante el consumo en adolescentes y profundizar en la relación entre las variables individuales y su implicación en la resistencia a la presión percibida para consumir alcohol, con la finalidad de trabajar sobre las variables citadas y desarrollarlas de manera favorable para reducir las altas tasas de consumo existentes entre la población adolescente. 
Asimismo, es preciso seguir con la línea de investigación y duplicar los esfuerzos en las estrategias de intervención innovadoras, proporcionando información provechosa que ofrezca un futuro mejor a esta población.

\section{AGRADECIMIENTOS}

El presente trabajo se ha desarrollado gracias al Proyecto Violencia entre iguales y consumo de alcohol y tabaco en Educación Secundaria: programa basado en realidad aumentada para la detección e intervención (Referencia: EDU2017-88139-R), financiado por el Programa Estatal de Investigación, Desarrollo e Innovación Orientada a los Retos de la Sociedad, en el marco del Plan Estatal de Investigación Científica y Técnica y de Innovación, y la cofinanciación con Fondos Estructurales de la Unión Europea. Así como, gracias a la financiación Formación de Profesorado Universitario en Áreas Deficitarias, Ayudas Gerty Cori, por la ayuda para la contratación de personal investigador en formación predoctoral, concedida a María del Mar Simón Márquez, y gracias a las ayudas para la formación de profesorado universitario de los subprogramas de Formación y Movilidad dentro del Programa Estatal de Promoción del Talento y su Empleabilidad, en el marco del Plan Estatal de Investigación Científica y Técnica y de Innovación 2017-2020 concedida a Rosa María del Pino Salvador (Referencia: FPU18/05857).

\section{BIBLIOGRAFÍA}

Adan, A. (2012). Impulsividad funcional y disfuncional en jóvenes con consumo intensivo de alcohol (binge drinking). Adicciones, 24(1), 17-22.

Álvarez, A., Alonso, M.M. y Guidorizzi, A.C. (2010). Consumo de alcohol y autoestima en adolescentes. Revista Latino-Americana Enfermagem, 18, 634-640.

Arias, A., Vargas, M. y Herazo, E. (2013). Confidencialidad y dimencionalidad del audit en estudiantes de medicina. Psicología desde el Caribe, 30(1), 21-35.

Calafat, A., García, F., Juan, M., Becoña, E. y Fernández-Hermida, J.R. (2014). Which parenting style is more protective against adolescent substance use? Evidence within the European context. Drug and Alcohol Dependence, 138, 185-192.

https://doi.org/10.1016/j.drugalcdep.2014.02.705

Cohen, J. (1988). Statistical Power Analysis for the Behavioral Sciences. Second Edition. Hillsdate, NJ: LEA.

Committee on National Alcohol Policy and Action (CNAPA). (2014). Action plan on youth drinking and on heavy episodic drinking. Recuperado de:

https://ec.europa.eu/health//sites/health/files/alcohol/docs/2014_2016_actionplan_youthdri nking_en.pdf

Contreras, L., Molina, V. y Cano, M.C. (2012). Consumo de drogas en adolescentes con conductas infractoras: análisis de variables psicosociales implicadas. Adicciones, 24(1), 31 38.

Cosi, S., Vigil-Colet, A., Canals, J. y Lorenzo-Seva, U. (2008). Psychometric properties of the spanish adaptation of Barratt Impulsiveness Scale-11-A for children. Psychological Reports, 103(2), 336-346. https://doi.org/10.2466/pr0.103.2.336-346 
Delegación del Gobierno para el Plan Nacional Sobre Drogas (2018). Encuesta estatal sobre uso de drogas en enseñanzas secundarias (ESTUDES) 1994/2016. Madrid: Secretaría General de Política Social y Consumo. Ministerio de Sanidad y Política Social.

Fuentes, M.C., Alarcón, A., García, F. y Gracia, E. (2015). Consumo de alcohol, tabaco, cannabis y otras drogas en la adolescencia: efectos de la familia y el barrio. Anales de Psicología, 31(3), 1.000-1.007.

Gázquez, J.J., Pérez-Fuentes, M.C., Molero, M.M., Martos, Á., Cardila, F., Barragán, A.B. y Mercader, I. (2015). Adaptación Española del Cuestionario de Expectativas del Alcohol en Adolescentes. European Journal of Investigation in Health, Psychology and Education, 5(3), 357-369. https://doi.org/10.1989/ejihpe.v5i3.138

Gázquez, J.J., Pérez-Fuentes, M.C., Carrión, J.J. y Santiuste, V. (2009). Estudio y análisis de conductas violentas en Educación Secundaria en España. Universitas Psichologica, 9(2), 371-380.

González-Yubero, S., Palomera, R. y Lázaro-Visa, S. (2019). Trait and ability emotional intelligence as predictors of alcohol consumption in adolescents. Psicothema, 31(3), 292297.

Heimisdottir, J., Vilhjalmsson, R., Kristjansdottir, G. y Meyrowitsch, D.W. (2010). The social context of drunkenness in mid-adolescence. Scandinavian Journal of Public Health, 38, 291-298. https://doi.org/10.1177/14034948093 57094

IBM Corp. Released 2015. IBM SPSS Statistics for Windows, Version 23.0. Armonk, NY: IBM Corp.

Jiménez, T. (2011). Autoestima de riesgo y protección: una mediación entre el clima familiar y el consumo de sustancias en adolescentes. Psychosocial Intervention, 20(1), 53-61.

Koob, G.F. y Volkow, N.D. (2010). Neurocircuitry of addiction. Neuropsychopharmacology Reviews, 35, 217-238.

Llorens, N., Barrio, G., Sánchez, A., Suelves, J.M. y ESTUDES Grupo de Trabajo. (2011). Effects of socialization and family factors on adolescent excessive drinking in Spain. Prevention Science, 12, 150-161. https://doi.org/10.1007/s11121-010-0195-0

Londoño, C., Valencia, S.C., Sánchez, L. y León, V. (2007). Diseño del cuestionario resistencia a la presión de grupo en el consumo de alcohol (CRPG). Suma Psicológica, 14(2), 271-288.

Londoño, C. y Valencia, S. (2010). Resistencia de la presión de grupo, creencias acerca del consumo y consumo de alcohol en universitarios. Anales de Psicología, 26(1), 27-33.

Maganto, C., Peris, M. y Sánchez, R. (2019). El bienestar psicológico en la adolescencia: variables psicológicas asociadas y predictoras. European Journal of Education and Psychology, 12(2), 139-151.

Martínez-Loredo, V., Fernández-Hermida, J.R., Fernández-Artamendi, S., Carballo, J.L. y García-Rodríguez, O. (2015). Spanish adaptation and validation of the Barratt Impulsiveness Scale for early adolescents (BIS-11-A). International Journal of Clinical and Health Psychology, 15, 274-282. https://doi.org/10.1016/j.ijchp.2015.07.002

Molina, J., Sandín, B. y Chorot, P. (2013). Sensibilidad a la ansiedad y presión psicológica: efectos sobre el rendimiento deportivo en adolescentes. Cuadernos de Psicología del Deporte, 14(1), 45-54.

Moñino, M., Piñero, E., Arense, J. y Cerezo, F. (2013). Violencia escolar y consumo de alcohol y tabaco en estudiantes deEducación Secundaria. European Journal of Investigation in Health,Psychology and Education, 3(2), 137-147.

Moral, M.V., Rodríguez, F.J. y Sirvent, C. (2006). Factores relacionados con las actitudes juveniles hacia el consumo de alcohol y otras sustancias psicoactivas. Psicothema, 18(1), 52-58.

Narváez, D.A. y Caro, E.J. (2015). Impulsividad funcional y disfuncional en adolescentes consumidores de alcohol. Revista Electrónica de Psicología Iztacala, 18(2), 539-563. 
Pedreira, J.L., Blanco, V., Pérez-Chacón, M.M. y Quirós, S. (2014). Psicopatología en la adolescencia. Medicine, 11(61), 3.612-3.621. https://doi.org/10.1016/S03045412(14)70821-2

Pérez-Fuentes, M.C., Gázquez, J.J., Molero, M.M., Cardila, F., Martos, A., Barragán, A.B. ... y Mercader, I. (2015). Impulsividad y consumo de alcohol y tabaco en adolescentes. European Journal of Investigation in Health, Psychology and Education, 5(3), 371-382.

Pérez-Fuentes, M.C., Molero, M.M. y Gázquez, J.J. (2019). Expectations and sensations-seeking as predictors of bringe drinking in adolescents. Annals of Psychology, 35(1), 124-130.

Pérez-Fuentes, M.C. y Gázquez, J.J. (2010). Variables relacionadas con la conducta violenta en la escuela según los estudiantes. International Journal of Psychology and Psychological Therapy, 10(3), 427-437.

Pérez-Gramaje, A. F., García, O. F., Reyes, M., Serra, E. y García, F. (2019). Parenting Styles and Aggressive Adolescents: Relationships with Self-esteem and Personal Maladjustment. The European Journal of Psychology Applied to Legal Context, 12(1), 1-10. https://doi.org/10.5093/ejpalc2020a1

Peterson, R.A., y Reiss, R.J. (1992). Anxiety Sensitivity Index Manual, 2nd ed. Worthington: International Diagnostic Systems.

Plan Nacional Sobre Drogas (2016). Encuesta sobre uso de drogas en enseñanzas secundarias en España (ESTUDES), 1994-2014. España: Ministerio de Sanidad Servicios Sociales e Igualdad.

Pons, J. y Buelga, S. (2011). Factores asociados al Consumo Juvenil de Alcohol: Una Revisión desde una Perspectiva Psicosocial y Ecológica. Psychosocial Intervention, 20(1), 75-94.

Riaño, D., Guillén, A. y Buela, G. (2015). Conceptualización y evaluación de la impulsividad en adolescentes: una revisión sistemática. Universitas Psychologica, 14(3), 1.077-1.090.

Ribas, M., del Prado, N., Claramunt, J., Civit, M., Canalias, O. y Santaolalla, A., (2015). Adolescentes multiproblemáticos: consumo de tóxicos y trastorno mental en jóvenes que delinquen. Actas Españolas de Psiquiatría, 43(6), 197-204.

Riquelme, M., García, O.F. y Serra, E. (2018). Desajuste psicosocial en la adolescencia: socialización parental, autoestima y uso de sustancias. Anales de Psicología, 34(3), 536544.

Rodríguez, M.C., Perozo, C.M. y Matute, J.D. (2014). Resistencia de la presión de grupo y consumo de alcohol en adolescentes. Revista de Psicología, 6(1), 25-40.

Rosenberg, M. (1965). Society and the adolescent self-image. Princeton, N.J: Princeton University Press.

Sanabria, A.M. y Uribe, A.F. (2009). Conductas antisociales y delictivas en adolescentes infractores y no infractores. Pensamiento Psicológico, 6(13), 203-218.

Sánchez, L. (2009). La sensibilidad a la ansiedad disminuye tras realizar un programa de enfermería en relajación. Revista Enfermería CyL, 1(1), 66-76.

Sandín, B., Valiente, R.M., Chorot, P. y Santed, M.A. (2007). ASI-3: Nueva escala para la evaluación de la sensibilidad a la ansiedad. Revista de Psicopatología y Psicología Clínica, 12(2), 91-104.

Teixidó-Compañó, E., Sordo, L., Bosque, M., Puigcorbe, S., Barrio, G., Brugal, M.T. ...Espelt, A. (2019). Factores individuales y contextuales relacionados con el binge drinking en adolescentes españoles: un enfoque multinivel. Adicciones, 31(1), 41-51.

Valdez, E. y Lira, J. (2013). Asociación entre la sensibilidad a la ansiedad y el consumo de tabaco. Enseñanza e Investigación en Psicología, 18(2), 343-358.

Veiga, F.H., García, F., Reeve, J., Wentzel, K. y García, O. (2015). When adolescents with high self-concept lose their engagement in school. Revista de Psicodidáctica, 20, 305-320. https://doi.org/10.1387/RevPsicodidact.12671 